SCHIAVONI, Martín. Recensión: NIÑO, Luis Fernando y MATUS ACUÑA, Jean Pierre, Dogmática jurídica y ejercicio del poder. Riesgos del vasallaje cultural en la doctrina penal latinoamericana; Buenos Aires, Editorial Astrea, 2017, 139 páginas.

Polít. crim. Vol. 13, № 25 (Julio 2018) Rec. 1, pp. 650 - 656.

[http://www.politicacriminal.cl/Vol_13/n_25/Vol13N25R1.pdf]

\title{
Recensión: NIÑO, Luis Fernando y MATUS ACUÑA, Jean Pierre, Dogmática jurídica y ejercicio del poder. Riesgos del vasallaje cultural en la doctrina penal latinoamericana; Buenos Aires, Editorial Astrea, 2017, 139 páginas.
}

\section{Review: NIÑO, Luis Fernando y MATUS ACUÑA, Jean Pierre, Dogmática jurídica y ejercicio del poder. Riesgos del vasallaje cultural en la doctrina penal latinoamericana; Buenos Aires, Editorial Astrea, 2017, 139 pages.}

\section{Martín Andrés Schiavoni ${ }^{1}$}

Tal como ya se anticipa desde el título, el libro comentado es Dogmática jurídica y ejercicio del poder; aunque lo que resulta más atrayente aún -por lo menos para el suscripto- es el subtítulo, ya que la inserción de la expresión riesgos del vasallaje cultural involucran conceptos, ideas y hasta sentimientos o emociones que exceden el árido, álgido y, en ocasiones, casi ininteligible ámbito de la nuda dogmática jurídica penal.

Y, como también surge nítidamente desde el propio título, se trata de una obra escrita a "cuatro manos". En principio, ya que a poco que ingresemos en su lectura, podemos corregir la afirmación y sostener que fueron "seis las manos" que participaron en su factura, ya que el extenso prólogo de Eugenio Raúl Zaffaroni no va a la saga, en interés y erudición, a los dos artículos que componen la obra.

Efectivamente, en el proemio al libro, el ex miembro de la Corte Suprema de Justicia argentina realiza mucho más que una mera presentación, ya que, a la par de desgranar los aspectos centrales de los dos opúsculos de la obra, introduce nuevos datos, conocimientos y afirmaciones propias que indudablemente enriquecen en forma notable la calidad del libro.

Como ya se adelantó, la obra se compone por dos artículos. El primero de ello, titulado ¿Derecho penal del enemigo en el marco del estado de derecho? corresponde al profesor de la Facultad de Derecho de la Universidad de Buenos Aires, Luis Fernando Niño, y -tal como se adivina fácilmente- se trata de una nueva reflexión en torno a la discutidísima cuestión del derecho penal del enemigo.

Tal como lo refiere Zaffaroni en su prólogo, la primera consideración acerca de la mencionada cuestión, por parte del profesor de la Universidad de Bonn, Günther Jakobs, “desató una catarata de escritos polémicos y se llevó muchísimos árboles de nuestros

\footnotetext{
${ }^{1}$ Magister en Criminología; Especialista en derecho procesal penal; especialista en derecho penal; todos por la Facultad de Derecho y Ciencias Sociales de la Universidad Nacional del Litoral. Correo electrónico: maschiavoni@hotmail.com
} 
Polít. crim. Vol. 13, No 25 (Julio 2018) Rec. 1, pp. 650 - 656.

[http://www.politicacriminal.cl/Vol_13/n_25/Vol13N25R1.pdf]

bosques". 2 Pues bien, una gota más en la siempre renovada catarata la constituye la presente contribución.

Entre las varias aristas sugestivas que ofrece la monografía de Niño, nos interesa destacar el repaso histórico-filosófico que desarrolla el autor, en su búsqueda y explicación de la cíclica distinción entre "propios y extraños, amigos y enemigos, ciudadanos y extranjeros, hospes u hostis". ${ }^{3}$ Dentro de dicho derrotero, el profesor argentino se concentra en algunos nombres clásicos, comenzando por Platón y Aristóteles, pasando por Tomás de Aquino, Hobbes, Rosseau, Fichte y Kant, para finalizar deteniéndose, con especial énfasis, en las opiniones del jurista y teórico político alemán Carl Schmitt y en las repercusiones que tales juicios -fundamentalmente en lo referente a la distinción dicotómica amigo-enemigo- encontraron durante el período nazi.

Avanzando más acá en el tiempo, Niño se adentra en la citada antinomia en el período moderno, a partir de su llegada e incorporación a la doctrina penal alemana contemporánea. Con cita en un conocido opúsculo de Muñoz Conde, nuestro autor fecha dicho advenimiento en 1985, en el marco de unas jornadas de penalistas alemanes desarrolladas en la ciudad de Frankfurt, ocasión en que el prestigioso profesor de la Universidad de Bonn, Günther Jakobs, brinda una conferencia e introduce su famoso derecho penal del enemigo, concepto que, con el paso de los años y a través de diferentes obras y disertaciones, es especificado, reelaborado y sistematizado.

Las reflexiones de Niño con respecto al derecho penal del enemigo resultan sumamente críticas; posición que, cabe aclarar, es la mayoritaria, por lo menos en la doctrina jurídica penal de este lado del Atlántico. Pero, además, las diatribas de nuestro autor son numerosas y diversas, las que aquí solamente serán enunciadas, ante la imposibilidad de introducirnos en ellas con detenimiento.

Así, Niño se focaliza -controvirtiendo algunos de tales tópicos- en las fuentes filosóficopolíticas del derecho penal del enemigo; para luego avanzar en la distinción entre personas en derecho y sujetos de derecho, lo que, a criterio del profesor argentino, entraña un desalojo del estatus jurídico del individuo.

A continuación, ausculta los riesgos e incompatibilidades que posee el derecho penal del enemigo, tanto con las Cartas Magnas modernas como con los diversos tratados

2 ZAFFARONI, Eugenio Raúl, "Prólogo", en: NIÑO, Luis Fernando y MATUS ACUÑA, Jean Pierre, Dogmática jurídica y ejercicio del poder. Riesgos del vasallaje cultural en la doctrina penal latinoamericana; Buenos Aires, Editorial Astrea, 2017, p. XI. La afirmación de Zaffaroni es absolutamente real. A fines meramente ilustrativos, nos vamos a limitar al siguiente ejemplo: en el año 2006 los profesores de la Universidad Autónoma de Madrid, Cancio Melia y Gómez-Jara Díez, coordinaron la publicación de dos enormes volúmenes, compuestos por artículos que se dedican, desde diversos prismas, exclusivamente a esta temática; específicamente, el volumen I contiene 30 monografías, mientras que el volumen II posee 40 artículos. La suma de páginas de ambos tomos ascienda a la singular cantidad de 2284 (CANCIO MELIA, Manuel y GÓMEZ-JARA DÍEZ, Carlos: Derecho Penal del Enemigo. El discurso penal de la exclusión; V. I y II; coeditado por Edisofer y Editorial BdeF, Buenos Aires, 2006).

3 NIÑO, Luis Fernando: “¿Derecho penal del enemigo en el marco del estado de derecho?”, en: NIÑO y MATUS ACUÑA, Dogmática jurídica, cita nota ${ }^{\circ} 2$, p. 1. 
SCHIAVONI, Martín. Recensión: NIÑO, Luis Fernando y MATUS ACUÑA, Jean Pierre, Dogmática jurídica y ejercicio del poder. Riesgos del vasallaje cultural en la doctrina penal latinoamericana; Buenos Aires, Editorial Astrea, 2017, 139 páginas.

internacionales de Derechos Humanos, en tanto implica el abandono del criterio del comienzo de ejecución de una acción delictiva, con la posible caída en un derecho penal de autor-acompañando tales críticas con ejemplos de las nefandas consecuencias que ello produjo en estados totalitarios-. En igual sendero, también advierte los peligros que conlleva responder en términos bélicos a los actos terroristas, entre otras varias cuestiones que no mencionamos por cuestiones de espacio.

En síntesis: por revisitar el pensamiento de algunos célebres cultores de la dicotomía amigo-enemigo; por examinar la cercanía de los fundamentos de Jakobs a fuentes contrarias al derecho penal liberal y por intersecar en su análisis voces provenientes de otros ámbitos disciplinarios -tales como las del filósofo y sociólogo Theodor Adorno o la del escritor Günther Grass- la de Niño constituye una aproximación más a una temática que, no por manida, deja de poseer una importancia y actualidad que obliga a repensarla constantemente.

El segundo de los artículos se intitula Por qué citamos a los alemanes y otros apuntes metodológicos y pertenece al profesor chileno de las Universidades de Chile y Finis Terrae, Jean Pierre Matus Acuña. Este trabajo es, en realidad, una nueva difusión -ahora revisado y ampliado- del texto que fuera originalmente publicado en el año 2008, a partir de una estancia de investigación que su autor realizó en la Universidad de Gotinga (Alemania), durante ese mismo año.

El nodo entre ambos trabajos es fácilmente advertible, sin necesidad de escrutar demasiado: el artículo de Niño analiza las consecuencias que tuvo -y tiene- la incorporación, al interior del derecho penal, de la concepción de la persona en tanto enemigo; construcción que, tal como se especificó, tuvo como mentor al profesor teutón Günther Jakobs. Pues bien, cualquier latinoamericano que se haya acercado a las aguas de la dogmática jurídica penal, así sea para tan solo nadar superficialmente en las mismas, habrá percibido la avasallante importancia que poseen los germánicos en tales ámbitos. Justamente lo que plantea Matus Acuña en su opúsculo es porqué esto es así y las implicancias que tiene esta "entusiasta recepción” en nuestra región, fundamentalmente en Chile y Argentina ${ }^{4}$.

En primer lugar, el profesor chileno realiza una breve aproximación histórica de dicha acogida en los países antes mencionados, para luego dar paso a la tentativa de explicación de los motivos o causales de dicha recepción.

En su recorrido, Matus Acuña analiza tres posibles respuestas: inicialmente, expresa que ello es plausible en tanto la dogmática de origen alemán se ubica en la cima del desarrollo científico en tal materia, lo que posibilitaría una aplicación más segura, racional, coherente y previsible de la ley penal por parte de los tribunales. En segundo lugar, porque la dogmática jurídica penal de dicho país es "liberal” y, por lo tanto, puede actuar como dique

${ }^{4}$ Con respecto a esta entusiasta recepción que tuvo la dogmática jurídica penal en Argentina, con especial énfasis en las labores de Luis Jiménez de Asúa, Sebastián Soler y Ricardo Nuñez, v. SCHIAVONI, Martín: Positivismo criminológico, dogmática jurídica penal y enseñanza universitaria; Córdoba, Editorial Brujas, 2017. 
Polít. crim. Vol. 13, No 25 (Julio 2018) Rec. 1, pp. 650 - 656.

[http://www.politicacriminal.cl/Vol_13/n_25/Vol13N25R1.pdf]

de contención a los conatos autoritarios. Por último, el autor ensaya una tercera respuesta: el dominio de tales conocimientos brinda a sus cultores la posibilidad de disponer y proponer a los operadores jurídicos locales propuestas normativas dogmáticamente justificadas y adaptables o moldeables a los cambios en las valoraciones sociales, a la par de generar una "fuente de autoridad" que bloquea el acceso de los legos a las discusiones sobre el contenido y alcance del derecho penal.

Variados resultan los tópicos interesantes y destacables del trabajo de Matus Acuña. Particularmente, queremos resaltar dos de ellos que, a nuestro criterio, son los más atrayentes: en primer lugar, el artículo del profesor chileno se asienta sobre una plataforma bibliográfica extraordinariamente sólida y extensa; basta repasar tanto la sección correspondiente a la bibliografía como así también las 115 notas a pie de página que posee el trabajo para advertir dicha solidez ${ }^{5}$. Mientras que, por otra parte, cada una de las tres posibles respuestas a la pregunta sobre "por qué citamos tanto a los alemanes" es objetada por el propio autor e, incluso, en ocasiones esa objeción también es refutada, con lo cual el autor termina generando un interesante juego de espejos, ahondando en la búsqueda de respuestas plausibles a sus interrogantes.

Finalmente, la obra de Matus Acuña posee otras dos cuestiones que la valorizan. Por un lado, a lo largo del parágrafo $\S 7$, titulado Colofón, el profesor chileno despliega una extensa respuesta a una "réplica" a su trabajo, efectuada por el profesor Alex Van Weezel'.

El propio Matus se ha encargado de sintetizar y refutar, en su Colofón, los tres "equívocos"7 que le achaca Van Weezel. En este sentido, la primera contraposición gira en torno al objeto de estudio de la ciencia del derecho penal, ya que Van Weezel le reprocha poseer "un equivocado concepto de ciencia penal", ya que Matus pretendería introducir, al interior de la ciencia del derecho penal, cuestiones absolutamente ajenas, tales como las características personales de los jueces, su origen social, sus filiaciones científicas y otras, en pos de tratar de predecir el contenido de sus sentencias; temáticas que, según Van Weezel, más allá de su importancia, no constituyen el objeto de estudio de la ciencia del derecho penal. A ello responde Matus que lo que se pretendió fue intentar comprender el propio sistema jurídico [es decir, el chileno], explorando las regularidades e irregularidades de la jurisprudencia, aprovechando para ello todos los aportes disponibles, sin limitarse "disciplinadamente" a los autores alemanes. Además, sobre este punto conflictivo, Matus agrega que considera sumamente relevante analizar las condiciones necesarias para la realidad del derecho al momento de verificar tales regularidades o irregularidades ${ }^{8}$.

\footnotetext{
${ }^{5} \mathrm{Si}$ bien es cierto que no necesariamente una extensa cantidad de bibliografía brinda seguridades acerca de la calidad de un trabajo de estas características, a nuestro criterio, en este caso la profusa bibliografía va acompañada de un exhaustivo análisis.

6 Específicamente, hacemos referencia a la siguiente publicación: VAN WEEZEL, Alex: “¿Por qué no citamos más (por ejemplo, a los alemanes)? Réplica a J. P. Matus"; en Política Criminal, n 6, 2008, pp. 1-5.

7 Ésta es, textualmente, la palabra utilizada por Van Weezel (VAN WEEZEL, "Por qué no citamos más", cita nota $\left.\mathrm{n}^{\circ} 6, \mathrm{p} .1\right)$.

${ }^{8}$ MATUS ACUÑA, Jean Pierre: "Por qué citamos a los alemanes y otros apuntes metodológicos", en: NIÑO y MATUS ACUÑA, Dogmática jurídica, cita nota n 2, pp. 101-103.
} 
SCHIAVONI, Martín. Recensión: NIÑO, Luis Fernando y MATUS ACUÑA, Jean Pierre, Dogmática jurídica y ejercicio del poder. Riesgos del vasallaje cultural en la doctrina penal latinoamericana; Buenos Aires, Editorial Astrea, 2017, 139 páginas.

En segundo lugar, la discusión avanza en torno a "una errónea comprensión de las relaciones entre lo universal y lo particular", controversia que pivotea justamente sobre la universalidad de determinados presupuestos de la ciencia jurídica penal y su vinculación con el derecho positivo. Específicamente, Van Weezel le reprocha a Matus referir que "las teorías germanas de la ciencia penal habrían adquirido universalidad al asumir presupuestos generales no sujetos a discusión y, al mismo tiempo, a costa de su vinculación con el derecho positivo". Y, siendo esto así, como a mayor universalidad menor posibilidad de aplicar las teorías sobre un sistema jurídico de un país concreto, tales teorías serían ignoradas en la práctica o bien cada operador jurídico seleccionaría una conforme lo que le conviene. Pero, además de discrepar sobre lo anterior, Van Weezel también discute la afirmación de Matus acerca de que este defecto sería consustancial a la ciencia penal alemana producto de su ambiciosa estrategia ${ }^{9}$. Sobre el tópico, Matus argumenta que lo que él efectuó fue una descripción de un estado de cosas y no una valoración sobre el mismo ${ }^{10}$. Por último, Matus replica la crítica de Van Weezel, quien le recrimina "poner de cabeza el proceso de formación del conocimiento", al entender que, según Matus, "lo primero es la cita", cuando en realidad lo primero es el problema a resolver, el que es abordado por el jurista a través del método propio de esta ciencia; las citas que aparecen en el derrotero en pos de obtener la solución más plausible, sólo son una muestra de honestidad intelectual del jurista o investigador ${ }^{11}$. Sobre esta contraposición, entiende Matus que Van Weezel ha incurrido en una interpretación equivocada de lo afirmado por él en el texto, ya que nunca desmereció la importancia del método científico alemán. Lo que sí hizo es criticar el nudo uso (y abuso) de la cita de autores alemanes, como argumento de autoridad, toda vez que ello excluye de la palestra la verdadera discusión y análisis. Agregando que, en lugar de limitarse a las "citas alemanas", habría que maximizar las posibilidades de análisis de los problemas jurídicos a partir de una verdadera visión de derecho comparado ${ }^{12}$.

Indudablemente que la lectura de los tres opúsculos -es decir, el inicial de Matus, la "réplica" de Van Weezel y la contrarréplica de Matus- enriquecen notablemente el debate en torno a la cuestión, permitiendo a los lectores visibilizar las aristas problemáticas y las posiciones contradictorias que existen sobre el tema ${ }^{13}$. Si bien, según expone Matus en su respuesta final, en el fondo dichas aristas y contradicciones no serían tales, sino tan solo "malos entendidos", de cualquiera manera estimamos que nos encontramos ante una interesante confrontación de posiciones, cuyo resultado final es un enriquecedor debate.

La segunda cuestión atrayente tiene que ver con los dos apéndices incluidos al final del artículo, mediante los cuales, a partir de dos interesantes análisis exploratorios, se intenta y a nuestro criterio se logra- demostrar estadísticamente su aseveración acerca de que los

\footnotetext{
9 VAN WEEZEL, "Por qué no citamos más", cita nota n ${ }^{\circ}$ 6, pp. 2-3.

10 MATUS ACUÑA: "Por qué citamos a los alemanes", cita nota n ${ }^{\circ}$ 8, p. 103-104.

${ }^{11}$ VAN WEEZEL, "Por qué no citamos más", cita nota ${ }^{\circ}$ 6, p. 4.

12 MATUS ACUÑA: "Por qué citamos a los alemanes", cita nota n ${ }^{\circ}$ 8, p. 104-107, passim.

${ }^{13}$ Las que aquí solo hemos podido enunciar de manera muy sucinta.
} 
Polít. crim. Vol. 13, No 25 (Julio 2018) Rec. 1, pp. 650 - 656.

[http://www.politicacriminal.cl/Vol_13/n_25/Vol13N25R1.pdf]

autores iberoamericanos utilizan copiosamente la bibliografía germana, mientras que ello no ocurre, ni remotamente, a la inversa.

En esencia, entre las varias virtudes que enumeramos más arriba, el trabajo de Matus Acuña resulta un atractivo aporte a la discusión acerca de la utilización -y sus demasías o excesosde las importaciones culturas de las zonas hegemónicas y su impacto y efectos en nuestra periférica. 
SCHIAVONI, Martín. Recensión: NIÑO, Luis Fernando y MATUS ACUÑA, Jean Pierre, Dogmática jurídica y ejercicio del poder. Riesgos del vasallaje cultural en la doctrina penal latinoamericana; Buenos Aires, Editorial Astrea, 2017, 139 páginas.

\section{Bibliografía}

CANCIO MELIA, Manuel y GÓMEZ-JARA DÍEZ, Carlos (2006): Derecho Penal del Enemigo. El discurso penal de la exclusión; V. I y II; coeditado por Edisofer y Editorial BdeF, Buenos Aires.

SCHIAVONI, Martín (2017): Positivismo criminológico, dogmática jurídica penal y enseñanza universitaria; Córdoba, Editorial Brujas.

WEEZEL, Alex van (2008): “¿Por qué no citamos más (por ejemplo, a los alemanes)? Réplica a J. P. Matus"; en Política Criminal, n 6, 2008, D2-6. 